\title{
Comparative RNA-seq analysis of the drought sensitive lentil (Lens culinaris) root and leaf under short- and long- term water deficits
}

Hande Morgil $^{1 \uparrow}$, Mehmet Tardu ${ }^{2 \uparrow}$, Gül Cevahir ${ }^{1}$, İbrahim Halil Kavakli²,3*

${ }^{1}$ Department of Biology, Istanbul University, 34134 Suleymaniye, Istanbul, Turkey

${ }^{2}$ Department of Chemical and Biological Engineering, Koc University, Rumelifeneri Yolu, Sariyer,

Istanbul, Turkey

${ }^{3}$ Department of Molecular Biology and Genetics, Koc University, Rumelifeneri Yolu, Sariyer, Istanbul, Turkey

ๆ these authors are equally contributed

*Corresponding Author

e-mail:hkavakli@ku.edu.tr

Tel: +902123381708

Keywords: Lentil, drought stress, roots, leaf, de novo RNA-seq 


\begin{abstract}
Drought stress is one of the main environmental factors that effects growth and productivity of crop plants, including lentil. To gain insights into the genome-wide transcriptional regulation in lentil root and leaf under short- and long-term drought conditions, we performed RNA-seq on a drought sensitive lentil cultivar (Lens culinaris Medik. cv. Sultan). After establishing drought conditions, lentil, samples were subjected to de novo RNA-seq based transcriptome analysis. The 207,076 gene transcripts were successfully constructed by de novo assembly from the sequences obtained from root, leaf, and stems. Differentially expressed gene (DEG) analysis on these transcripts indicated that period of drought stress had a greater impact on the transcriptional regulation in lentil root. The numbers of DEGs were 2,915 under short-term drought stress while the numbers of DEGs were increased to 18,327 under long-term drought stress condition in the root. Further, Gene Ontology analysis revealed that following biological processes were differentially regulated in response to long-term drought stress: protein phosphorylation, embryo development seed dormancy, DNA replication, and maintenance of root meristem identity. Additionally, DEGs, play role in circadian rhythm and photoreception, were down-regulated suggesting that drought stress has a negative effect on the internal oscillators which may have detrimental consequences on plant growth and survival. Collectively, this study provides a detailed comparative transcriptome response of drought sensitive lentil strain under short- and long-term drought conditions in root and leaf. Our finding suggests that not only the regulation of genes in leaves are important but also genes regulated in roots are important and needs to be considered for improving drought tolerance in lentil.
\end{abstract}




\section{INTRODUCTION}

Drought is one of the major environmental factors that inhibit the growth and productivity of crop plants. Studies with different plants showed that drought stress result in up to $50 \%$ yield losses (Daryanto et al. 2016; Wang et al. 2003). In the near future, global climate change is likely to gradually increase the intensity of drought which may result in even more yield losses (Battisti and Naylor 2009;Dai 2013). Therefore, there is a need to understand which mechanisms by which crop plants are affected by drought stress, and how they can tolerate it.

Several studies have been conducted on various crop plants to understand the effect of drought stress at physiological, molecular and biochemical levels (Lamaoui et al. 2018). Plants exhibit morphological changes such as enhanced root formation, decreased leaf area, reduced stomata number and its conductance, increased leaf thickness, and leaf rolling to minimize water loss and maximizing the water uptake (Sicher et al. 2012). As drought conditions persist, plant produces wax to cover aerial part of it to minimize dehydration (Lee and Suh 2013). At biochemical level, plants produce osmolytes like proline and glycine betaine to protect various components of cell to minimize the effect of water loss (Lamaoui, et al. 2018). Genomics approaches such as microarray and RNA-seq are used to understand genome-wide regulation in various crop plants at the molecular level, and to relate with physiological and biochemical changes. Studies revealed a complex interaction network between different transcription factors to enable plants to produce the appropriate response. In addition, genomics studies also highlighted the importance 
of transcriptional plasticity among different cultivars of the same crop plants (Kulkarni et al. 2017), which adds another layer of complexity and necessities further genome-wide studies.

Lentil is one of the legume crop plants whose productivity is affected by drought stress causing 6-54\% yield loss (Johansen et al. 1994;Oweis et al. 2004;Sehgal et al. 2017; Stoddard et al. 2006). Several breeding programs have been initiated to obtain drought tolerant by breeding different genotype of the lentil lines (Singh et al. 2016). Recently, a genome-wide study has been performed on leaf tissues of drought-sensitive and resistance lentil lines to identify the affected biological processes (Singh et al. 2017). Currently, it is still not known how drought stress effects transcriptional regulation in different tissues of lentil under short- and long-term droughtstress conditions. Additionally, it is not known how transcriptional plasticity occurs in lentil depending on its genotype under short- and long-term drought conditions. Therefore, we took a genome-wide approach to understand the effect of drought period on transcriptional response and plasticity using drought-sensitive lentil (Lens culinaris Medik. cv. Sultan) root and leaf tissues. After de novo transcriptome assembly using RNA-seq reads, differentially expressed gene (DEG) analysis showed a drastic global transcriptional change occur in root when compared with leaf under long-term drought stress. The GO enrichment analysis of the DEGs revealed that genes involved in transcription, regulation of, transcription, response to abscisic acid, protein phosphorylation and protein ubiquitination were commonly regulated in first day stress conditions in both root and leaf. However, as stress prolonged the expression of additional genes playing role in processes of light response, circadian rhythm, and positive regulation of cell death were affected. Additionally, as duration of drought stress increased several genes having role in root development DNA repair, cell cycle DNA replication, sterol biosynthetic process, mismatch 
repair, cell death response to salt stress, tyrosine kinase signaling pathways, and mitotic nuclear division were highly regulated in root tissues.

Our study indicated the complexity of lentil plant responses to short- and long-term drought stress in different tissues. These data will help us to understand mechanisms to drought response, may be used to improve productivity and yield of plants under drought stress.

\section{MATERIALS AND METHODS}

\section{Determination of Optimum Drought Condition}

Seeds of lentil (Lens culinaris Medik. cv. Sultan) were kindly provided by the Southeastern Anatolia Agricultural Research Institute of Turkey. The seeds were selected for size homogeneity, surface-sterilized for $2-3 \mathrm{~min}$ in $1 \%$ sodium hypochlorite, rinsed and soaked in distilled water. Seeds were then placed in $6 \mathrm{~cm}$ petri dishes and germinated by watering with 5 $\mathrm{ml}$ of $\mathrm{dH}_{2} \mathrm{O}$ containing $0 \%$ (control), $5 \%, 20 \%$, and $25 \%$ polyethylene glycol (PEG) (MW 6000) concentration corresponding to final osmotic potentials of $0.0,-0.10,-0.14$, and $-0.19 \mathrm{MPa}$, respectively. PEG, constitute water stress and lack of penetration to plant cells, was used to adjust osmotic potential and also exhibit no toxic effects to plant cells. The petri dishes were then sealed with parafilm to prevent evaporation and they were kept in a controlled climate chamber at a temperature of $25^{\circ} \mathrm{C} / 18^{\circ} \mathrm{C}$ day/night; photoperiod was $16 / 8 \mathrm{~h}$ light/dark with $10 \%$ humidity. Seeds were regarded "germinated" when the radicle had extended for at least $3 \mathrm{~mm}$. After 7 days of treatment, the 10 -seeds in the control group ( $0 \%$ PEG) were completely germinated, drought dose were determined for the seedling survivability or medium with different concentrations of PEG. Seedling survivability percent was calculated as follows: 
Seedling survivability $\%=100 \mathrm{x}$ (number of seedlings which survived under the drought stress) / (total number of seedlings) (Idrissi et al. 2016). The data was obtained as three biological replicates with 10 seeds.

\section{Hydroponic Assay and Drought-stress Treatment}

Seeds were surface-sterilized for $5 \mathrm{~min}$ in $1 \%$ sodium hypochlorite and rinsed with $\mathrm{dH}_{2} \mathrm{O}$ and then germinated on perlit medium with irrigated everyday by $50 \mathrm{ml}$ Hoagland's nutrient solution $\left(5 \mathrm{mM} \mathrm{KNO}_{3}, 5 \mathrm{mM} \mathrm{Ca}\left(\mathrm{NO}_{3}\right)_{2}, 1 \mathrm{mM} \mathrm{MgSO} 4,5 \mathrm{mM} \mathrm{KH}_{2} \mathrm{PO}_{4}, 0.1 \mathrm{mM}\right.$ Fe-EDTA, $46 \mathrm{mM}$ $\left.\mathrm{H}_{3} \mathrm{BO}_{3}, 4.5 \mathrm{mM} \mathrm{MnCl}_{2}, 3.8 \mathrm{mM} \mathrm{ZnSO}_{4}, 0.3 \mathrm{mM} \mathrm{CuSO}_{4}, 0.1 \mathrm{mM} \mathrm{NH}_{4} \mathrm{MoO}_{7}\right)$. Seven days old seedlings were transferred to hydroponic medium (Conn et al. 2013). Control samples were kept in the Hoagland's nutrient solution for the entire hydroponic assay, the drought stress exposed samples were kept in the Hoagland's nutrient solution including 15\% PEG 6000, for 5 days. All the experimental pods were aerated through the experiment by air pump and kept in a controlled climate chamber at a temperature of $25^{\circ} \mathrm{C} / 18^{\circ} \mathrm{C}$ day/night; photoperiod was $16 / 8 \mathrm{~h}$ light/dark with $10 \%$ humidity.

\section{Physiological Analysis}

Samples were harvested at $24^{\text {th }}$ hours $\left(1^{\text {st }}\right.$ day) and $96^{\text {th }}$ hours $\left(4^{\text {th }}\right.$ day) after drought stress treatment in hydroponic medium. Following physiological parameters were determined as described in (Kabbadj et al. 2017): relative water content (RWC), total chlorophyll content, proline content, and lipid peroxidation.

The measurements of the chlorophyll fluorescence were carried out from lentil samples by Imaging-PAM Chlorophyll Fluorometer (Walz Effeltrich, Germany) as previously described in 
(Duan et al. 2016). Briefly, sample plants were kept in the dark for $10 \mathrm{~min}$ and then exposed to the weak-measuring ray of the fluorescence imaging instrument where the minimal level of fluorescence $\left(F_{o}\right)$ was obtained due to the open state of the photosystem II (PSII) centers. Then samples were exposed to a saturating light pulse with a photosynthetic photon flux density (PPFD) of $960 \mu \mathrm{mol}$ photons $\mathrm{m}^{-2} \mathrm{~s}^{-1}$ for $0.5 \mathrm{~s}$. Under this conditions PSII centers will go into closed state which resulted in the maximal level of fluorescence $\left(F_{m}\right)$. After high-light exposure the fluorescence level were expected to decrease to a stable state. After that sample leaves were exposed to an actinic PPFD of $260 \mu \mathrm{mol}$ photons $\mathrm{m}^{-2} \mathrm{~s}^{-1}$ to be able to calculate the transient fluorescence level, $F$, before a peak value of fluorescence, $F_{p}$. The following parameters were calculated: $F_{v} / F_{m}$, фPSII, $\phi N P Q, \phi N O, q P$ and ETR (Baker 2008;Barbagallo et al. 2003;Kramer et al. 2004) .

\section{Preparation of cDNA Libraries for RNA-seq}

Total RNA was isolated from $1 \mathrm{gr}$ of root, stem and leaves of lentil grown in hydroponic culture as described above (see section Hydroponic Assay and Drought-stress Treatment), using TRIzol reagent (Invitrogen, USA) as described in (Sarayloo et al. 2017). BioAnalyzer (Agilent, USA) was used to measure the quality and the quantity of the isolated total RNAs. After that, total RNA samples were treated with RNase-free DNase I (Thermo Scientific, USA) at a concentration of $1 \mathrm{U} / \mu \mathrm{g}$ to eliminate the genomic DNA. Then, RNA-seq library preparation was performed using TruSeq Stranded RNA LT Kit (Illumina, USA) as previously described in (Lahens et al. 2014;Tardu et al. 2017; Tardu et al. 2016). The 100x2 paired-end sequencing of cDNA libraries for control and drought stress exposed samples $\left(1^{\text {st }}\right.$ day and $4^{\text {th }}$ day samples of root and leaf, and $1^{\text {st }}$ day sample of stem) with two biological replicates were performed using 
the Illumina HiSeq4000 sequencing platform (Illumina, USA).

\section{De novo Transcriptome Assembly and Quality Assessment}

The quality of the raw reads were checked using FastQC tool

(http://www.bioinformatics.babraham.ac.uk/projects /fastqc/) (v0.11.5) and then Trimmomatic (v0.35) (Bolger et al. 2014) was used to remove the reads with adaptor contamination and lowquality reads (phred score $<20$ ) with the argument -sliding window 4:20. The high quality reads were saved in fastq files and deposited in the GEO database at NCBI with the accession number: GSE115199. All of the downstream analyses were done using the high quality, clean data. De novo assembly was performed using Trinity tool (http://trinityrnaseq.sourceforge.net/) with the default parameters (Grabherr et al. 2011). To construct a de novo transcriptome assembly for lentil, we used all drought stress exposed samples together with their controls (no-stress samples)

at the indicated time points for root and leaf $\left(1^{\text {st }}\right.$ day and $4^{\text {th }}$ day samples $)$, and stem tissues $\left(1^{\text {st }}\right.$ day sample, which its RNA seq data were only used for de novo assembly to obtain increase coverage. No further analysis was carried out on $1^{\text {st }}$ day stem samples. After constructing de novo transcriptome assembly, the completeness of the assembly was assessed by CEGMA (Parra et al. 2007). Then, full-length transcript counting was done by BLASTX homology search $($ BLAST + v2.3.0) with the arguments -evalue 1e-20 -num_threads 6 -max_target_seqs 1 -outfmt 6 against the UniProt database and running perl script analyze_blastPlus_topHit_coverage.pl from the Trinity package.

\section{Differential Expression Analysis}

Reads were mapped to the de novo transcriptome assembly using Bowtie aligner (Langmead et al. 2009) with default parameters. Then, RSEM method (Li and Dewey 2011) was used to 
quantify transcript abundances in all samples. To assess the reproducibility between biological replicates of each sample, a Pearson's correlation analysis was applied to obtain the transcriptlevel $\mathrm{R}^{2}$ using RSEM-computed counts. For count-based differential expression testing between root and leaf tissues at different time-points, edgeR (Robinson et al. 2010) was used with the following arguments: min_rowSum_counts of 10 , false discovery rate $($ FDR $)<0.01$ and the value of $\mid \log _{2}$ fold change $\mid \geq 1$.

\section{Gene Ontology (GO) Assignments and KEGG Pathway annotations of the Transcripts in}

\section{the Assembly}

Annocript tool (Musacchia et al. 2015) was used to annotate trinity assembled de novo transcripts (https://github.com/frankMusacchia/Annocript/tree/master/GUIDE). We used the Swiss-Prot (SP) and UniRef90 (version: February 2016) for the blastp against proteins with the following arguments: word_size $=4$; e-value $=10^{-5}$; num_descriptions $=5$; num_alignments $=5$; threshold $=18$. For each sequence, the best hit was chosen. Rpsblast parameters, to identify domain composition of putative proteins in the Conserved Domains Database (CDD) (MarchlerBauer et al. 2015), were: e-value $=10^{-5}$; num_descriptions $=20$; num_alignments $=20$.

For gene ontology assignments, annotated de novo transcripts were analyzed using Database for Annotation, Visualization and Integrated Discovery (DAVID, https://david.ncifcrf.gov/) (Dennis et al. 2003) with default parameters. The statistical significance is determined using the Benjamini correction; adjusted $p<0.05$ was considered significant. In order to obtain KEGG Pathway annotations of the Transcripts in the Assembly, all the de novo assembled transcripts were aligned with GENES (http://www.genome.jp/kegg/genes.html) using BLAST algorithm (Blastx/Blastp 2.2.29+) based on KEGG database (http://www.genome.jp/kegg/). 


\section{Validation of RNA-seq Results Using qRT-PCR}

To validate the RNA-seq results, qRT-PCR was performed for 12 representative DEGs. In briefly, total RNA was extracted using TRIzol reagent (Invitrogen, USA) followed by DNase I treatment. Then, $1 \mu \mathrm{g}$ of total RNA was used for first-strand cDNA synthesis using random hexamer oligos. qRT-PCR was performed with gene-specific primers shown in Table S1 using Luminaris HiGreen qRT-PCR Master Mix (Thermo Scientific, USA) on the CFX Connect RealTime PCR Detection System (Biorad, USA). $\beta$-tubulin gene whose expression level didn't change across samples was used as an internal reference gene. The amplification programs were performed according to the following protocol: $95^{\circ} \mathrm{C}$ for $10 \mathrm{~min} ; 40$ cycles of $95^{\circ} \mathrm{C}$ for $15 \mathrm{~s}, 58$ ${ }^{\circ} \mathrm{C}$ for $30 \mathrm{~s}$ and $72{ }^{\circ} \mathrm{C}$ for $30 \mathrm{~s}$, and followed by a thermal denaturing step to generate the melting curves. All reactions were performed in biological triplicates (each triplicate with two technical replicates), and the results were expressed relative to the transcription level of $\beta$-tubulin gene in each sample by using the $2^{-\triangle \Delta C T}$ method (Livak and Schmittgen 2001). The mRNA expression data analyzed by using GraphPad Prism version 7 (GraphPad software, USA). All data were presented as the means \pm S.D. $(n=6)$ of the relative mRNA expression.

\section{RESULTS AND DISCUSSION}

\section{Seedling Response to Drought-stress and Physiological Analysis}

In this study, a drought-sensitive lentil cultivar (Lens culinaris Medik. cv. Sultan) was used to get better insight into the genome-wide transcriptional regulation of root and leaf tissues under short- and long-term drought conditions. For this purpose, lentil seeds were germinated at 
different concentrations of PEG to determine the optimum drought concentration by calculating the survival rate (Figure 1a). Seeds were grown in the presence of the 15\% PEG had survival rate of $60 \%$ compared to control $(p<0.0001)$. Then, leaf samples were harvested after 24 hours $\left(1^{\text {st }}\right.$ day; referred as short-term) and 96 hours $\left(4^{\text {th }}\right.$ day; referred as long-term) and were subjected to the biochemical and physiological analysis to determine the level of drought stress. The relative water content (RWC), total chlorophyll ( $C h l)$, proline, and malonyldialdehyde (MDA) contents were measured as drought stress indicators. The RWC values of the samples taken from the $1^{\text {st }}$ day $(p<0.01$ versus control $)$ and $4^{\text {th }}$ day $(p<0.005$ versus control $)$ were significantly lower than control (Figure 1b). Expectedly, the RWC value of the long-term drought exposed seedlings were $35 \%$ lower than the seedlings exposed to short-term drought stress. The analysis of the $C h l$ content of the samples indicated that there was significantly low $C h l$ content in samples under short- $(p<0.01$ versus control) and long-terms $(p<0.005$ versus control) drought stress (Figure 1c). Another indicator of the drought stress is the lipid peroxidation level, which is determined by the level of the MDA content. MDA levels were significantly higher in stressed samples compared to control (Figure 1d). Finally, the amount of proline was measured in the samples, which are expected to increase under drought stress. As seen in Figure 1e, proline contents were significantly increased in both $1^{\text {st }}$ day $\left(p<0.005\right.$ versus control) and $4^{\text {th }}$ day $(p<0.01$ versus control) drought-stress samples. All these results showed that drought stress had great impact on lentil.

\section{Chlorophyll Fluorescence Imaging Analysis}

The efficiency of photosystem II $\left(F_{v} / F_{m}\right)$, operating efficiency of photosystem II ( $\left.\Phi_{\mathrm{PSII}}\right)$, photochemical quenching (qP), and rate of photosynthetic electron transport (ETR) are expected to decrease while the efficiency of non-photochemical quenching $(\$ \mathrm{NPQ})$ and non-regulated 
energy dissipation (фNO) are expected to increase in plants under drought stress (Yao et al.

2018). Therefore, we measured all these parameters to ensure that the seedlings were under stress conditions. As expected, $F_{v} / F_{m}$, фPSII, and qP values were reduced under the drought conditions

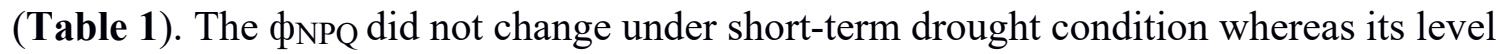
increased significantly under long-term drought condition $(p<0.05)$. We estimated the fraction of open PSII reaction centers by measuring with modulated fluorescence qP. Results showed that long-term drought stress caused a significant decrease $(\sim 40 \%, p<0.01)$ in the number of open PSII reaction centers when compared to the short-term drought stress (Table 1). Other photosynthetic parameters, ETR and \$NO, were also monitored. The ETR decreased during the continued drought and showed a significant decline at the end of the $4^{\text {th }}$ day $(p<0.01)$. We also observed increased $\phi_{N O}$ levels after long-term drought stress which indicates an increase the plant's inability to cope with radiation (Table 1).

Collectively, all physiological data suggested that the lentil seedlings were in drought conditions and RNA-seq study was conducted using leaves and root samples for understanding the effect of short- and long drought stress in lentil at transcrit level.

\section{Sequencing and De novo Assembly Statistics}

After collecting samples from seedlings of lentil root, stem and leaf, grown in hydroponic conditions, total RNAs extraction was followed by mRNA isolation. Then, RNA-seq libraries were prepared and sequenced using Illumina platform. 
Sequencing resulted in $\sim 60 \mathrm{G}$ bp raw output. Before de novo transcriptome assembly of the samples with Trinity tool (http://trinityrnaseq.sourceforge.net/) (Zhao et al. 2011), raw data were subjected to analysis to eliminate low quality reads (phred score $<20$ ) and short reads with length $<20$ nucleotide. After filtering, a total of 568,571,434 paired-end high quality clean reads with an average length of 64 bp were obtained. De novo assembly generated 207,076 transcripts with a mean length of $950 \mathrm{nt}$. At the gene level, assembly resulted in 124,151 genes with N50 length of 1,638 nt (Table 2). Length distribution analyses of the transcripts revealed that 94,449 transcripts (45.61\%) have the length between 500 to 2,500 nts. Additionally, the shortest transcript has the $201 \mathrm{nts}$ whereas the longest transcript has $12,367 \mathrm{nts}$. When compared with other published data (Singh, et al. 2017; Sudheesh et al. 2016), the number of transcripts in our de novo transcriptome assembly was significantly higher possibly due to the usage of different de novo assemblers (Xie et al. 2014). Additionally, using RNA-seq data from different lentil tissues including root, stem and leaf both under short-term and long-term drought stress conditions may enable us to get a more complete de novo transcriptome assembly with a high number of transcripts.

\section{Evaluation of Quality and Accuracy of $L$. culinaris de novo Transcriptome Assembly}

CEGMA tool (Parra et al. 2007) was used to determine quality and correctness of de novo assembled transcriptome. This analysis revealed that the 246 out of 248 core eukaryotic proteins (corresponds to $99.2 \%$ coverage) were completely covered in the assembled transcriptome. Then, all reads were mapped back to assembled transcriptome using Bowtie (Langmead et al. 2009) to obtain mapping percentage of each RNA-seq sample. The $92-96 \%$ of all reads were successfully mapped back to the assembled transcriptome, which indicates correctness of the assembly and 
enabled us to perform further analysis. Additionally, full-length transcript analysis was performed to evaluate the quality of our transcriptome assembly. Results indicated that top matching proteins, which had at least $80 \%$ of their sequences included in the assembled transcripts, composed of 54\% $(9,394 / 17,574)$ of all matched proteins (Table S2).

To validate RNA-seq results, 12 DEGs from leaf and root samples, subjected to short- and longdrought stress, were randomly selected and their transcription levels were measured by qRTPCR. Both qRT-PCR and RNA-seq results exhibited similar transcriptional level for each gene, indicating that RNA-seq results were reliable and that the results obtained using both methods were comparable for all conditions (Figure S1).

\section{Functional Annotation of Trinity Assembled Transcripts}

Trinity tool was used to calculate the number of possible open reading frames (ORFs) of assembled transcripts (Grabherr, et al. 2011). Our results revealed that $72.8 \%$ of assembled transcripts $(150,680$ out of 207,076$)$ possess ORFs. Annocript pipeline was used to annotate these ORFs and to determine putative long non-coding RNAs (lncRNA) of the assembled transcripts (Musacchia, et al. 2015). This analysis identified 96,549 and 138,111 hits in the Swiss-prot and UniRef databases, respectively. Additionally, the 8730 possible lncRNAs $(p<0.05$ and maximum length of ORF: 100) were predicted. Identified transcripts of L. culinaris by de novo assembly displayed a significant similarity with those in Medicago truncatula (34.6\%), followed by those in Cicer arietinum (18.3\%) and Papilionoideae (12.9\%) (Figure 2 and Table S3). Our result is consistent with previously published results where they have shown high similarity between lentil and C.arietinum and M. truncatula (Singh, et al. 2017; Sudheesh, et 
al. 2016). Our result is different than the others where our analysis revealed that L. culinaris Medik. cv Sultan has the third highest homology with Papilionoideae (12.9\%).

\section{Gene Ontology Annotation and Kyoto Encyclopedia of Genes and Genomes Pathway Mapping}

In order to understand the biological significance of the transcripts in the transcriptome assembly, gene ontology (GO) term enrichment analysis was performed with DAVID tool (Dennis, et al. 2003). GO term assignments were used to classify de novo assembled transcripts into three categories: biological process (BP), molecular function (MF) and cellular component (CC). GO analysis for the 87,298 of L. culinaris transcripts (42.2\%) were performed, and GO assignments were plotted by WEGO (http://wego.genomics.org.cn/) (Figure 3). CC category contains 14 functional classes, classes for cell and cell part accounted for the major proportion with 54,248 (62.1\%) and 53,935 (61.8\%) transcripts, respectively. In the MF assignments, classes for catalytic activity with 44,114 transcripts (50.5\%) and binding with 44,003 transcripts $(50.3 \%)$ were mostly enriched. In the BP category, classes for cellular process and metabolic process possess highest number of transcripts, with cellular process $41,589(47.6 \%)$ and metabolic process 35,237 (40.4\%) transcripts, respectively. In addition, 11,692 (13.4\%) transcripts were classified under pigmentation and 9,824 (11.3\%) transcripts were classified under response to stimulus in the GO assignments.

To fully uncover the biological pathways that L. culinaris possess, all the assembled sequences were assigned with Kyoto Encyclopedia of Genes and Genomes (KEGG) orthology (KO) 
identifiers using KAAS (http://www.genome.jp/tools/kaas/) with the single-directional best hit method, and subsequently mapped to pathways using the KEGG API (http://www.kegg.jp/kegg/rest/keggapi.html). As a result, 25,829 transcripts were assigned to 399 different KEGG pathways (Table S4). The pathways with the highest number of unique transcripts were metabolic pathways ( 848 members), biosynthesis of secondary metabolites (389 members), biosynthesis of antibiotics (213 members), microbial metabolism in diverse environments (168 members), ribosome (127 members), carbon metabolism (107 members), biosynthesis of amino acids (107 members) and oxidative phosphorylation (104 members)

\section{(Figure 4).}

\section{Identification of Differentially Expressed Genes (DEGs) in Leaf and Root under Drought- Stress}

To identify the transcriptional changes at genome-wide level under short ( 24 hours, $1^{\text {st }}$ day) and long-term drought (96 hours, $4^{\text {th }}$ day) conditions in leaf and root tissues, DEG analyses have been performed. We, initially, carried out Pearson's correlation test to evaluate the reproducibility among biological replicates using the expression values (counts per million). After observing a strong correlation $\left(\mathrm{R}^{2}>0.95\right)$ between the biological replicates of each sample (Figure S2), edgeR tool was applied to identify the differentially expressed genes (DEGs) under different drought conditions in both tissues. All identified DEGs were given in the Table S5. In the $1^{\text {st }}$ day of drought condition, the transcriptional regulations were significantly altered in leaf tissues when compared with root tissues. The numbers of the DEGs were 6,949 in leaf while the numbers of the DEGs were 2,915 in the root (Figure 5a). Among these 2,725 and 1,745 genes 
were up-regulated whereas 4,224 and 1,170 genes were down-regulated in leaf and roots,

respectively (Figure 5a). A similar analysis was carried on the RNA-seq data exposed to longterm drought conditions. The numbers of DEGs in root were 18,327, which were remarkably high compared with number of DEGs under short-term drought condition (Figure 5A). On the other hand, the numbers of the DEGs in leaf were 8,306 , which were slightly higher compared with the number of DEGs identified under short-term drought stress. These data suggested that long-term drought stress in lentil had great impact on the gene regulation in root of lentil. Additionally, the comparison of numbers of DEGs in leaf and root samples $(6,949$ DEGs vs 2,915 DEGs) under short-term drought stress showed that leaf adapt to the drought stress more quickly than the root tissues in L. culinaris and produces a more rapid response at the transcriptional level against to water loss. Our results are consistent with the results of a previously reported study, where it was shown that there were twice the number of DEGs in the roots than in the leaves in peach, subjected to drought stress for 16 days (Ksouri et al. 2016). In addition, another study performed on a drought tolerance sunflower where plants were exposed to $24 \mathrm{~h}$ of drought stress in the presences of the $15 \%$ PEG 6000 revealed that the number of DEGs in leaf tissue is significantly higher than the root tissue after 24 hours drought condition (Liang et al. 2017).

\section{Comparison of DEGs between Root and Leaf under Short- and Long-Terms Drought}

\section{Conditions}

After the identification of DEGs for each sample, we determined common and sample specific DEGs in both tissues under two drought conditions (Figure 5b). The analysis showed that 195 
DEGs were commonly regulated in all condition and tissues. We performed a GO term enrichment analysis for 195 DEGs and identified significantly enriched biological processes and molecular function assignments (Figure 6a-c, Table S6). The most effected biological processes were regulation of transcription (19 DEGs), DNA-templated transcription (18 DEGs), response to abscisic acid (9 DEGs), and response to water deprivation (7 DEGs) (Figure 6a, Table S6). The root is the primary organ that responds at early stages of water deficit to mediated by abscisic acid (ABA) (Jiang and Hartung 2008). This may produce an immediate response through root to initiate $\mathrm{ABA}$ signaling which may result in root-to-shoot signaling and in the partial or complete stomatal closure to reduce transpiration. Therefore, ABA signaling pathway is heavily regulated throughout all drought conditions. At gene level, for example, a transcription factor called MYB108 was shown to be act as a negative regulator of ABA-induced cell death under biotic and abiotic stress in Arabidopsis (Mengiste et al. 2003). Another example, a receptor-like kinase FERONIA which plays a crucial role in plant response to small molecule hormones (e.g., auxin and ABA) (Chen et al. 2016). In addition, the most affected molecular function and cellular components were protein binding, transcription factor activity and plasma membrane, respectively (Figure 6a, Table S6). In fact, analysis of the most effected genes in each category indicated their role in drought stress specifically in ABA signaling in various studies (Shinozaki and Yamaguchi-Shinozaki 2007).

We then aimed to identify specific biological processes mediated by DEGs in root and leaf tissues under short- and long-term drought conditions. GO term enrichment analysis revealed 1,159 DEGs in root under both drought conditions (Figure 5b). The major significantly $(p<0.05)$ up-regulated biological processes were transcription, regulation of transcription, and protein 
ubiquitination while major significantly $(p<0.05)$ down-regulated biological processes were protein phosphorylation, transport, and tyrosine kinase signaling pathway under both short- and long term drought conditions in root (Figure 7a, Table S6). On the other hand, 1,699 DEGs were specifically expressed under short-term drought condition while 17,148 DEGs were expressed under the long-term drought condition (Figure 5b). It is surprising to see very high number of the DEGs in the $4^{\text {th }}$ day of the drought condition. To see how all these DEGs were distributed among the biological processes we again performed GO term enrichment analysis. As can be seen in Figure 7a, some of the up- and down-regulated processes (such as protein transport, response to ABA, RNA splicing, flower development, nitrate transport, sterol biosynthesis) in the $1^{\text {st }}$ day of drought condition were disappeared and instead the new biological process were seems to be up- and down-regulated in the $4^{\text {th }}$ day of drought condition (such as protein phosphorylation, mismatch repair, development of the seed dormancy, cell division, and DNA replication maintenance of the DNA repeat element). A very similar trend was observed in comparison of the DEGs between $1^{\text {st }}$ day and $4^{\text {th }}$ day leaf samples. The majority of the DEGs and biological processes (transcription, regulation of transcription, activated signaling pathway, and response to water deprivation) were common under both short- and long-term drought conditions (Figure 7a, Table S6). Additionally, some of the pathways were specifically regulated under long-term drought conditions. The following biological processes were up-regulated: response to cold, response to salt stress and ubiquitin-dependent protein degradation while these biological processes were down-regulated: chloroplast organization, circadian rhythm, positive regulation of cell death and cellular response to light stimulus (Figure 7a, Table S6). We also compared our results with a previously published result by Singh et al. (2017). Although our and their studies were carried out under different conditions, the genes that play role in the following 
processes were seems to be commonly regulated in the leaf: Response to abscisic acid DNAtemplated transcription, plant type cell wall, cell wall organization, chloroplast. The other affected processes were different from each other in both stdies. All these results suggested that different sets of gene were being regulated under different drought conditions, which may reflect transcriptional plasticity or experimental differences that performed under different conditions. Additionally, as drought stress persists the transcription of the genes are heavily regulated in roots when compared with leaf.

Previous studies highlight the importance of the circadian rhythm in drought stress (Legnaioli et al. 2009). Our results are consistent with previous studies where we showed circadian rhythms in both tissues are being most effective under either condition in the lentil. For example, one of the core clock proteins named as CRYPTOCHROMEs are a part of core clock protein in mammals (Kavakli et al. 2017) and function as the photoreceptors that sense environmental cues, such as irradiance, day-night transition, photoperiods, and light quality for optimal growth and development in plant (Liu et al. 2011). Also, Lysine-specific demethylase JMJ30, involved in the control of flowering time by demethylating $\mathrm{H} 3 \mathrm{~K} 36 \mathrm{me} 2$ at the FT locus and repressing its expression, acts within the central clock (Jones et al. 2010). In addition, a number of genes encodes circadian-associated factors have been regulated such as APRR7, REVEILLE 1 FBX2c, GIGANTEA, TOC2, and Photoropin-2 (Adams et al. 2015; Cha et al. 2017;Lu et al. 2017).

\section{CONCLUSION}

The current study reports the transcriptome analysis of leaves and roots related to short- and long-terms drought stress at the seedling stage in lentil using next generation sequencing approach. Due to lack of a reference genome for lentil, we first constructed a transcriptome 
assembly and then transcripts were annotated and mapped to the KEGG pathways. At the beginning of the drought stress, root and leaf produces very initial response by regulating the transcription of the several genes required to cope with drought stress. The persistence of the stress causes a huge transcriptional regulation at genome-wide level in the roots of the lentil. The

additional 11, 201 genes being up-regulated possibly regulate processes to minimize the effect of the stress in the root. All these results suggest that genes specifically express in roots which can be further utilized and serve as new resources for future genetic and functional genomics studies for drought tolerance in lentil.

\section{AUTHOR CONTRIBUTIONS}

HM and MT carried out experiments. MT performed analysis. MT, HM and GC contribute writing the manuscript. IHK designed experiments and writing the manuscript.

\section{FUNDING}

This work was supported by an Istanbul Development Agency grant (ISTKA-TR/14/EVK/0039) to IHK, by a Istanbul University BAP project $(21207,22583)$ to GC and IHK.

\section{ACKNOWLEDGEMENTS}

We would like to thanks Southeastern Anatolia Agricultural Research Institute providing us seed of Lens culinaris Medik. cv. Sultan.

\section{References}


Adams S, Manfield I, Stockley P, Carre IA (2015) Revised morning loops of the arabidopsis circadian clock based on analyses of direct regulatory interactions. Plos One 10.

Baker NR (2008) Chlorophyll fluorescence: a probe of photosynthesis in vivo. Annu Rev Plant Biol 59:89-113.

Barbagallo RP, Oxborough K, Pallett KE, Baker NR (2003) Rapid, noninvasive screening for perturbations of metabolism and plant growth using chlorophyll fluorescence imaging. Plant Physiol 132:485-493.

Battisti DS, Naylor RL (2009) Historical warnings of future food insecurity with unprecedented seasonal heat. Science 323:240-244.

Bolger AM, Lohse M, Usadel B (2014) Trimmomatic: a flexible trimmer for Illumina sequence data. Bioinformatics 30:2114-2120.

Cha JY, Kim J, Kim TS, Zeng Q, Wang L, Lee SY, Kim WY, Somers DE (2017) GIGANTEA is a co-chaperone which facilitates maturation of ZEITLUPE in the Arabidopsis circadian clock. Nat Commun 8.

Chen J, Yu F, Liu Y, Du CQ, Li XS, Zhu SR, Wang XC, Lan WZ, Rodriguez PL, Liu XM, Li DP, Chen LB, Luan S (2016) FERONIA interacts with ABI2-type phosphatases to facilitate signaling cross-talk between abscisic acid and RALF peptide in Arabidopsis. $P$ Natl Acad Sci USA 113:E5519-E5527.

Conn SJ, Hocking B, Dayod M, Xu B, Athman A, Henderson S, Aukett L, Conn V, Shearer MK, Fuentes S, Tyerman SD, Gilliham M (2013) Protocol: optimising hydroponic growth systems for nutritional and physiological analysis of Arabidopsis thaliana and other plants. Plant Methods 9:4.

Dai AG (2013) Increasing drought under global warming in observations and models. Nat Clim Change 3:52-58.

Daryanto S, Wang LX, Jacinthe PA (2016) Global synthesis of drought effects on maize and wheat production. Plos One 11.

Dennis G, Jr., Sherman BT, Hosack DA, Yang J, Gao W, Lane HC, Lempicki RA (2003) DAVID: Database for annotation, visualization, and integrated discovery. Genome Biol 4:P3.

Duan Z, Kong F, Zhang L, Li W, Zhang J, Peng L (2016) A bestrophin-like protein modulates the proton motive force across the thylakoid membrane in Arabidopsis. J Integr Plant Biol 58:848-858.

Grabherr MG, Haas BJ, Yassour M, Levin JZ, Thompson DA, Amit I, Adiconis X, Fan L, Raychowdhury R, Zeng QD, Chen ZH, Mauceli E, Hacohen N, Gnirke A, Rhind N, di Palma F, Birren BW, Nusbaum C, Lindblad-Toh K, Friedman N, Regev A (2011) Fulllength transcriptome assembly from RNA-Seq data without a reference genome. Nat Biotechnol 29:644-U130.

Idrissi O, Udupa SM, De Keyser E, McGee RJ, Coyne CJ, Saha GC, Muehlbauer FJ, Van Damme P, De Riek J (2016) Identification of quantitative trait loci controlling root and shoot traits associated with drought tolerance in a lentil (Lens culinaris Medik.) recombinant inbred line population. Front Plant Sci 7:1174.

Jiang F, Hartung W (2008) Long-distance signalling of abscisic acid (ABA): the factors regulating the intensity of the ABA signal. J Exp Bot 59:37-43.

Johansen C, Baldev B, Brouwer JB, Erskine W, Jermyn WA, Lijuan L, Malik BA, Miah AA, Silim SN (1994) Biotic and abiotic stress constraining productivity of cool-season food legumes in Asia, Africa and Oceania. Curr Plant Sci Biot 19:175-194.

Jones MA, Covington MF, DiTacchio L, Vollmers C, Panda S, Harmer SL (2010) Jumonji domain protein JMJD5 functions in both the plant and human circadian systems. P Natl Acad Sci USA 107:21623-21628. 
Kabbadj A, Makoudi B, Mouradi M, Pauly N, Frendo P, Ghoulam C (2017) Physiological and biochemical responses involved in water deficit tolerance of nitrogen-fixing Vicia faba. PLoS One 12:e0190284.

Kavakli IH, Baris I, Tardu M, Gul S, Oner H, Cal S, Bulut S, Yarparvar D, Berkel C, Ustaoglu P, Aydin C (2017) The Photolyase/Cryptochrome family of proteins as DNA repair enzymes and transcriptional repressors. Photochem Photobiol 93:93-103.

Kramer DM, Johnson G, Kiirats O, Edwards GE (2004) New Fluorescence Parameters for the Determination of QA Redox State and Excitation Energy Fluxes. Photosynth Res 79:209.

Ksouri N, Jimenez S, Wells CE, Contreras-Moreira B, Gogorcena Y (2016) Transcriptional responses in root and leaf of Prunus persica under drought stress using RNA sequencing. Front Plant Sci 7:1715.

Kulkarni M, Soolanayakanahally R, Ogawa S, Uga Y, Selvaraj MG, Kagale S (2017) Drought Response in Wheat: Key Genes and Regulatory Mechanisms Controlling Root System Architecture and Transpiration Efficiency. Front Chem 5:106.

Lahens NF, Kavakli IH, Zhang R, Hayer K, Black MB, Dueck H, Pizarro A, Kim J, Irizarry R, Thomas RS, Grant GR, Hogenesch JB (2014) IVT-seq reveals extreme bias in RNA sequencing. Genome Biol 15:R86.

Lamaoui M, Jemo M, Datla R, Bekkaoui F (2018) Heat and drought stresses in crops and approaches for their mitigation. Front Chem 6.

Langmead B, Trapnell C, Pop M, Salzberg SL (2009) Ultrafast and memory-efficient alignment of short DNA sequences to the human genome. Genome Biol 10:R25.

Lee SB, Suh MC (2013) Recent advances in cuticular wax biosynthesis and its regulation in Arabidopsis. Mol Plant 6:246-249.

Legnaioli T, Cuevas J, Mas P (2009) TOC1 functions as a molecular switch connecting the circadian clock with plant responses to drought. EMBO J 28:3745-3757.

Li B, Dewey CN (2011) RSEM: accurate transcript quantification from RNA-Seq data with or without a reference genome. BMC Bioinformatics 12.

Liang C, Wang W, Wang J, Ma J, Li C, Zhou F, Zhang S, Yu Y, Zhang L, Li W, Huang X (2017) Identification of differentially expressed genes in sunflower (Helianthus annuus) leaves and roots under drought stress by RNA sequencing. Bot Stud 58:42.

Liu H, Liu B, Zhao C, Pepper M, Lin C (2011) The action mechanisms of plant cryptochromes. Trends Plant Sci 16:684-691.

Livak KJ, Schmittgen TD (2001) Analysis of relative gene expression data using real-time quantitative PCR and the 2(T)(-Delta Delta C) method. Methods 25:402-408.

Lu H, McClung CR, Zhang C (2017) Tick tock: Circadian regulation of plant innate immunity. Annu Rev Phytopathol 55:287-311.

Marchler-Bauer A, Derbyshire MK, Gonzales NR, Lu S, Chitsaz F, Geer LY, Geer RC, He J, Gwadz M, Hurwitz DI, Lanczycki CJ, Lu F, Marchler GH, Song JS, Thanki N, Wang Z, Yamashita RA, Zhang D, Zheng C, Bryant SH (2015) CDD: NCBI's conserved domain database. Nucleic Acids Res 43:D222-226.

Mengiste T, Chen X, Salmeron J, Dietrich R (2003) The BOTRYTIS SUSCEPTIBLE1 gene encodes an R2R3MYB transcription factor protein that is required for biotic and abiotic stress responses in Arabidopsis. Plant Cell 15:2551-2565.

Musacchia F, Basu S, Petrosino G, Salvemini M, Sanges R (2015) Annocript: a flexible pipeline for the annotation of transcriptomes able to identify putative long noncoding RNAs. Bioinformatics 31:2199-2201.

Oweis T, Hachum A, Pala M (2004) Lentil production under supplemental irrigation in a Mediterranean environment. Agr Water Manage 68:251-265. 
Parra G, Bradnam K, Korf I (2007) CEGMA: a pipeline to accurately annotate core genes in eukaryotic genomes. Bioinformatics 23:1061-1067.

Robinson MD, McCarthy DJ, Smyth GK (2010) edgeR: a Bioconductor package for differential expression analysis of digital gene expression data. Bioinformatics 26:139-140.

Sarayloo E, Tardu M, Unlu YS, Simsek S, Cevahir G, Erkey C, Kavakli IH (2017) Understanding lipid metabolism in high-lipid-producing Chlorella vulgaris mutants at the genome-wide level. Algal Res 28:244-252.

Sehgal A, Sita K, Kumar J, Kumar S, Singh S, Siddique KHM, Nayyar H (2017) Effects of drought, heat and their interaction on the growth, yield and photosynthetic function of lentil (Lens culinaris Medikus) genotypes varying in heat and drought sensitivity. Front Plant Sci 8.

Shinozaki K, Yamaguchi-Shinozaki K (2007) Gene networks involved in drought stress response and tolerance. J Exp Bot 58:221-227.

Sicher RC, Timlin D, Bailey B (2012) Responses of growth and primary metabolism of waterstressed barley roots to rehydration. J Plant Physiol 169:686-695.

Singh D, Singh CK, Taunk J, Tomar RS, Chaturvedi AK, Gaikwad K, Pal M (2017) Transcriptome analysis of lentil (Lens culinaris Medikus) in response to seedling drought stress. BMC Genomics 18:206.

Singh D, Singh CK, Tomar RSS, Taunk J, Singh R, Maurya S, Chaturvedi AK, Pal M, Singh R, Dubey SK (2016) Molecular Assortment of Lens Species with Different Adaptations to Drought Conditions Using SSR Markers. Plos One 11.

Stoddard FL, Balko C, Erskine W, Khan HR, Link W, Sarker A (2006) Screening techniques and sources of resistance to abiotic stresses in cool-season food legumes. Euphytica 147:167-186.

Sudheesh S, Verma P, Forster JW, Cogan NO, Kaur S (2016) Generation and Characterisation of a Reference Transcriptome for Lentil (Lens culinaris Medik.). Int J Mol Sci 17.

Tardu M, Bulut S, Kavakli IH (2017) MerR and ChrR mediate blue light induced photo-oxidative stress response at the transcriptional level in Vibrio cholerae. Sci Rep 7:40817.

Tardu M, Dikbas UM, Baris I, Kavakli IH (2016) RNA-seq analysis of the transcriptional response to blue and red light in the extremophilic red alga, Cyanidioschyzon merolae. Funct Integr Genomic 16:657-669.

Wang W, Vinocur B, Altman A (2003) Plant responses to drought, salinity and extreme temperatures: towards genetic engineering for stress tolerance. Planta 218:1-14.

Xie Y, Wu G, Tang J, Luo R, Patterson J, Liu S, Huang W, He G, Gu S, Li S, Zhou X, Lam TW, Li Y, Xu X, Wong GK, Wang J (2014) SOAPdenovo-Trans: de novo transcriptome assembly with short RNA-Seq reads. Bioinformatics 30:1660-1666.

Yao JN, Sun DW, Cen HY, Xu HX, Weng HY, Yuan F, He Y (2018) Phenotyping of Arabidopsis drought stress response using kinetic chlorophyll fluorescence and multicolor fluorescence imaging. Front Plant Sci 9: 603

Zhao QY, Wang Y, Kong YM, Luo D, Li X, Hao P (2011) Optimizing de novo transcriptome assembly from short-read RNA-Seq data: a comparative study. BMC Bioinformatics 12 Suppl 14:S2. 
Table 1. Effect of drought stress (DS) on the chlorophyll fluorescence parameters $\left(F_{v} / F_{m}\right)$ and changes in the balance between light capture and energy use in L. culinaris seedlings.

\begin{tabular}{lllllll}
\hline Sample & $\mathbf{F}_{\mathbf{v}} / \mathbf{F}_{\mathbf{m}}$ & $\mathbf{\Phi}_{\mathbf{P S I I}}$ & $\mathbf{\Phi}_{\mathbf{N P Q}}$ & $\mathbf{\Phi}_{\mathbf{N O}}$ & $\mathbf{q P}$ & $\mathbf{E T R}$ \\
\hline $1^{\text {st }}$ day control & $0,81 \pm 0,01$ & $0,42 \pm 0,02$ & $0,22 \pm 0,02$ & $0,26 \pm 0,01$ & $0,75 \pm 0,01$ & $57,58 \pm 2,10$ \\
\hline $1^{\text {st }}$ day DS & $0,79 \pm 0,01 *$ & $0,39 \pm 0,01$ & $0,24 \pm 0,04$ & $0,34 \pm 0,09$ & $0,56 \pm 0,02$ & $49,62 \pm 1,15$ \\
\hline $4^{\text {th }}$ day control & $0,82 \pm 0,00$ & $0,41 \pm 0,01$ & $0,23 \pm 0,04$ & $0,27 \pm 0,00$ & $0,79 \pm 0,05$ & $51,37 \pm 2,35$ \\
\hline $4^{\text {th }}$ day DS & $0,58 \pm 0,03 * *$ & $0,19 \pm 0,02 *$ & $0,32 \pm 0,02^{*}$ & $0,57 \pm 0,04 * *$ & $0,31 \pm 0,04 * *$ & $25 \pm 1,85^{* *}$ \\
\hline
\end{tabular}

All measurements are represented as mean values \pm standard deviation of three biological replicates. Tukey test was performed to test for significant differences. An asterisk represents significantly different means of the same parameter between control and drought-stress samples. $(* p<0.05),(* * p<0.01)$. 
Table 2. Sequencing and assembly statistics of Lens culinaris Medik. cv. Sultan transcriptome.

\begin{tabular}{lr}
\hline \multicolumn{1}{c}{ Item } & Value \\
\hline Total number of clean reads after filtering & $568,571,434$ \\
\hline Mean length of trimmed read pairs (nt) & 64 \\
\hline Total number of assembled transcripts & 207,076 \\
\hline Assembled transcripts size in Mbp & 196,5 \\
\hline Mean transcript length (nt) & 950 \\
\hline Median contig length (nt) & 550 \\
\hline N50 value of transcripts (nt) & 1,638 \\
\hline Longest transcript length (nt) & 12,367 \\
\hline Total number of genes & 124,151 \\
\hline
\end{tabular}




\section{Figure Legends}

Figure 1. (A) L. culinaris seedling survivability percentage under different drought-stress conditions ( $0 \%$ (control), 15\%, 20\% and 25\% PEG). Errors bars represent standard deviation (SD) $(n=30)$. Physiological analysis of $L$. culinaris leaves with respect to changes in (B) relative water content, $(\mathbf{C})$ total chlorophyll $(C h l)$ content, (D) malondialdehyde content, and (E) proline content in control (no-stress) and drought-stress (+) samples. Assays were done after 24 hours $\left(1^{\text {th }}\right.$ day) and 96 hours ( $4^{\text {th }}$ day) of stress treatment. Means, standard errors of three biological and three technical replicates. The data were analyzed using a two-way ANOVA analysis of variance (Brown-Forsythe Test). Asterisks indicate significant differences at $* \mathrm{p}<0.05,{ }^{*} \mathrm{p}<0.01$, $* * * \mathrm{p}<0.005$ versus control samples.

Figure 2: UniProt Top-Hit species distribution of transcripts.

Figure 3: Gene ontology (GO) functional classification of transcripts in L. culinaris. The visualization of GO distribution was done with WEGO tool (http://wego. genomics.org.cn/) using GO level 2 descriptions for three main categories (biological process, molecular function, cellular component).

Figure 4: The top 10 assigned KEGG pathways with the highest transcript numbers. Numbers in the pie-chart represent the number of transcripts in the assigned KEGG pathways.

Figure 5: (A) Number of differentially expressed genes (DEGs) under drought-stress in leaf and root samples of $L$. culinaris at $1^{\text {st }}$ and $4^{\text {th }}$ days. (B) Venn diagram shows the number of DEGs across leaf and root tissues after $1^{\text {st }}$ and $4^{\text {th }}$ days of drought-stress and the overlap between each set of genes.

Figure 6: Significantly enriched $(p<0.05)$ GO term analysis of common 195 DEGs; (A) biological processes, (B) molecular functions and (C) cellular compartments.

Figure 7: Significantly enriched gene ontology (GO) biological processes (BP) in leaf and root tissues of L. culinaris. Each panel shows significantly enriched GO biological processes in (A) $1^{\text {st }}$ day and $4^{\text {th }}$ day samples in leaf, and (B) $1^{\text {st }}$ day and $4^{\text {th }}$ day samples of root. Asterisks indicate significantly regulated BP terms both in $1^{\text {st }}$ day and $4^{\text {th }}$ day samples. Number of enriched genes for each BP terms are represented both with blue and red colors. Down-regulated genes were represented with blue color whereas up-regulated genes were represented with red color. 


\section{SUPPLEMENTARY MATERIAL}

Table S1: qRT-PCR forward and reverse primer sequences.

Table S2: Transcripts were aligned to Uniprot protein database using BlastX

Table S3: Species distribution Medicago truncatula

Table S4: KEGG pathways

Table S5: Identified DEGs in all samples.

Table S6: Significantly enriched gene ontology (GO) biological process terms. 
a

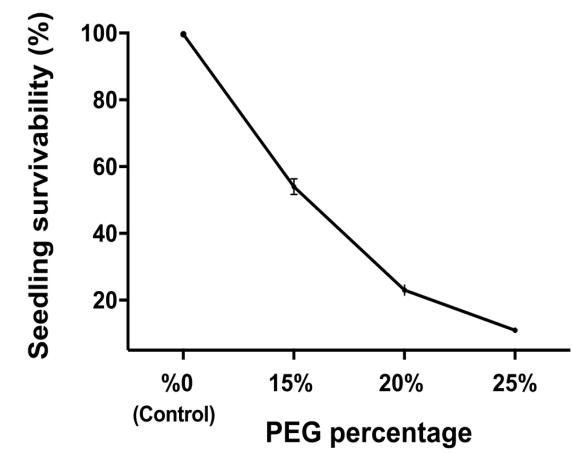

d

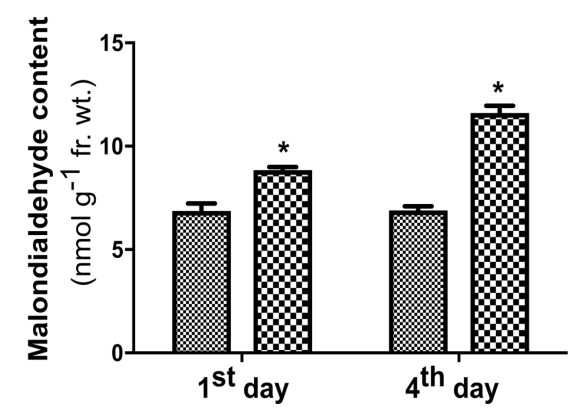

b

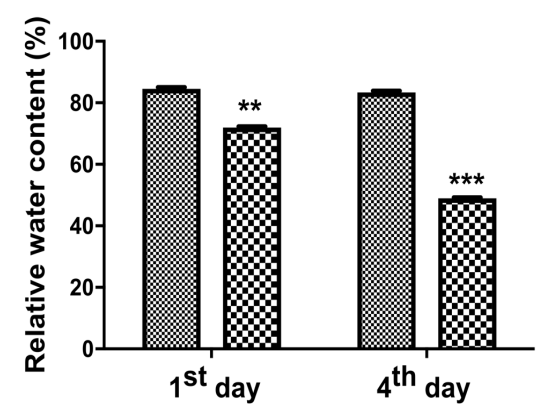

e

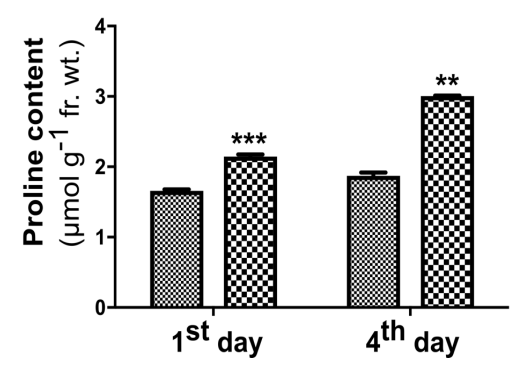

C

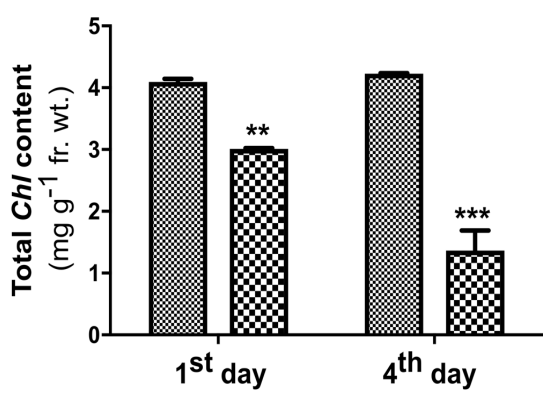

\& Control (no-stress)

Drought-stress (+)

Figure 1 


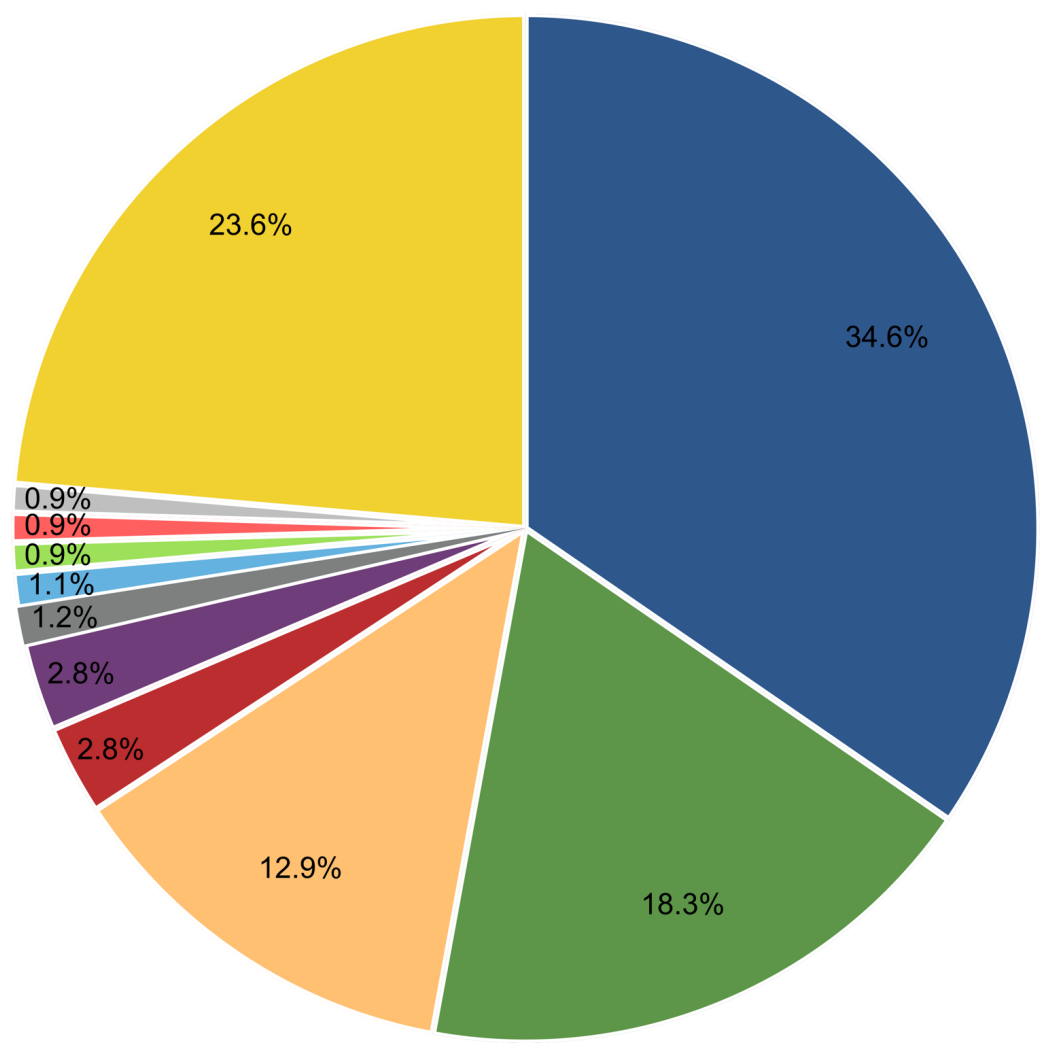

- Medicago truncatula

- Cicer arietinum

Papilionoideae

- Glycine max

- Fusarium

- Pisum sativum

- Phaseoleae

- Rhizopus delemar

- Glycine soja

- Soja

Others

Figure 2 


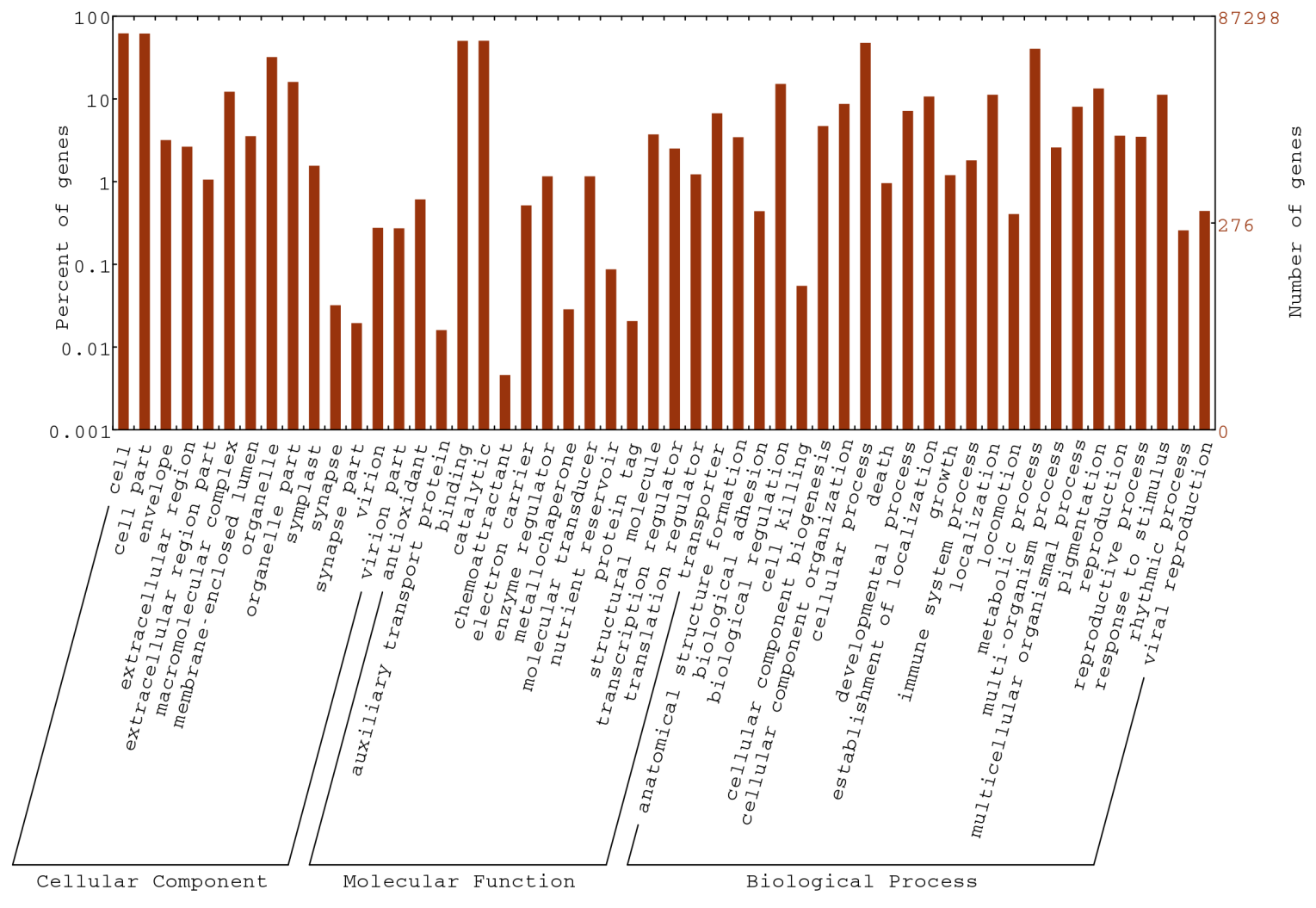

Figure 3 


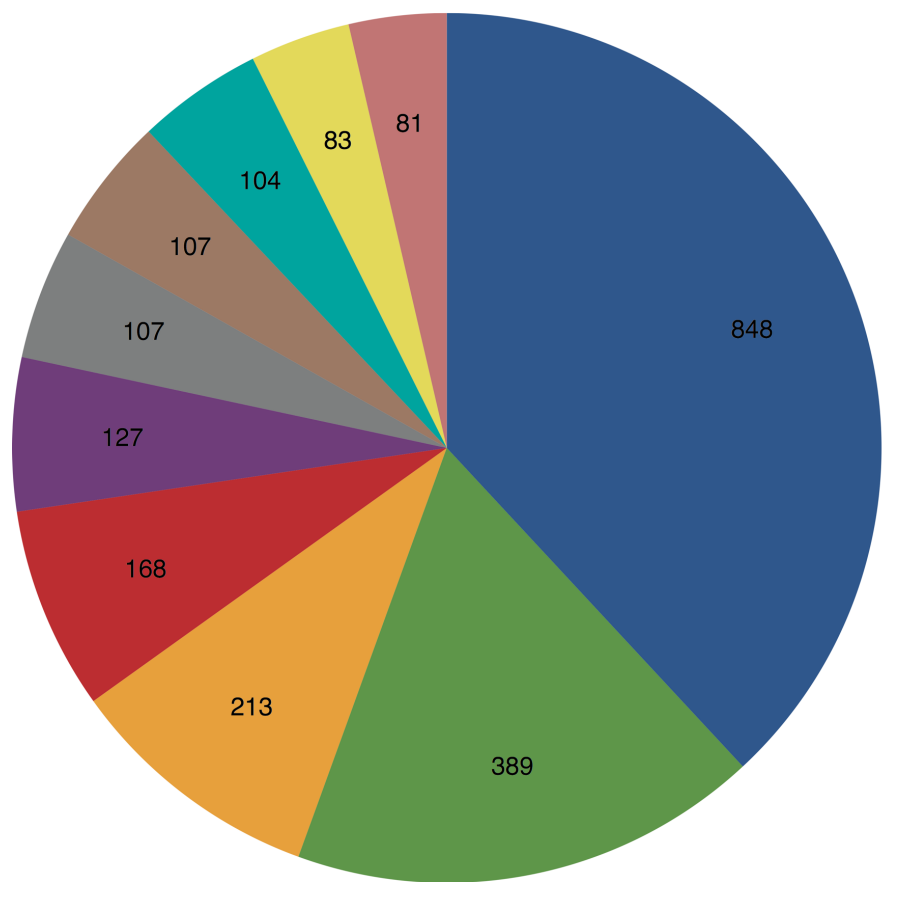

- Metabolic pathways

- Biosynthesis of secondary metabolites

- Biosynthesis of antibiotics

- Microbial metabolism in diverse environments

- Ribosome

- Carbon metabolism

- Biosynthesis of amino acids

- Oxidative phosphorylation

Spliceosome

- Protein processing in endoplasmic reticulum

Figure 4 
a

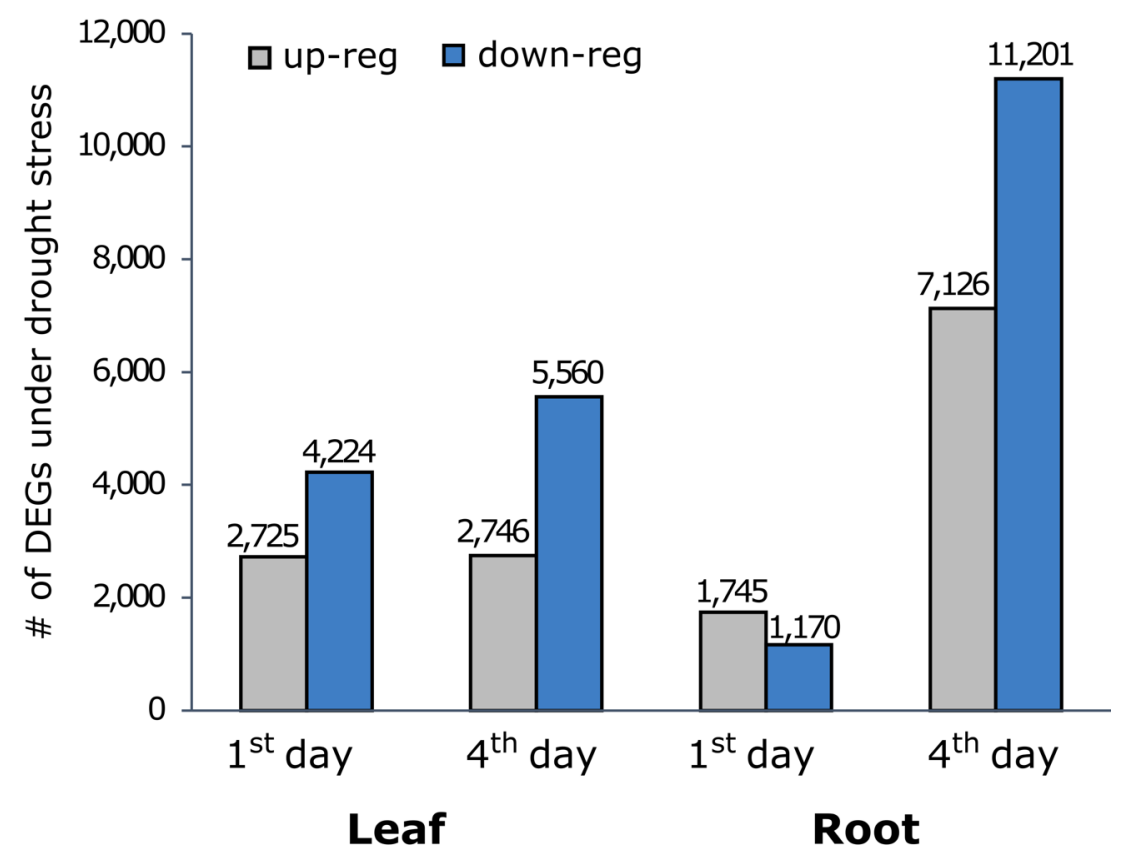

b

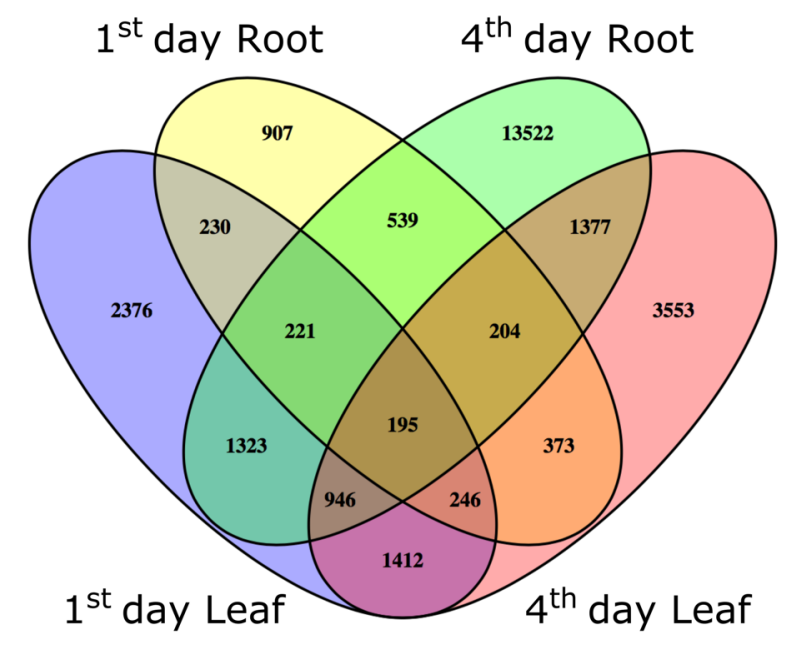

Figure 5 


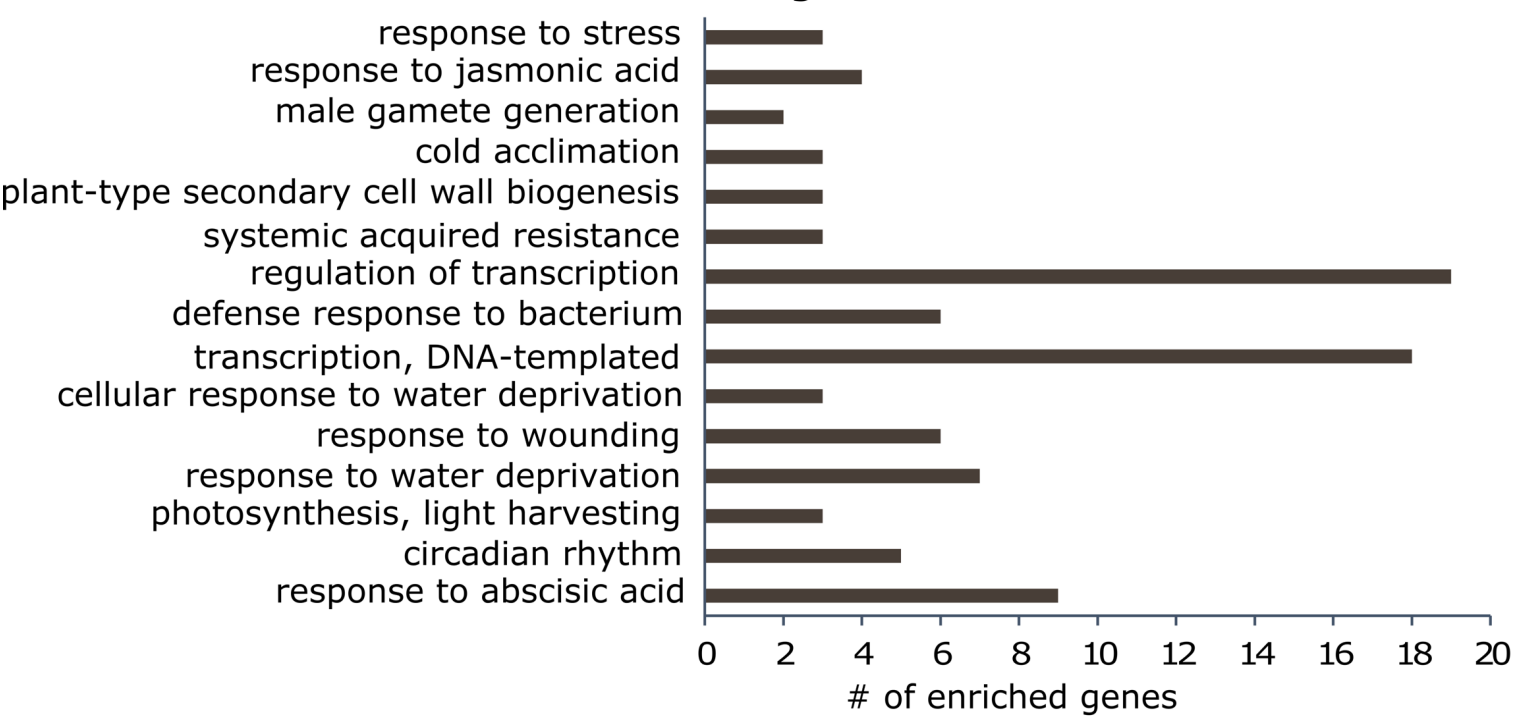

b

\section{Common Molecular Function}

kinase activity sequence-spesific DNA binding protein self-association transcription factor activity protein binding

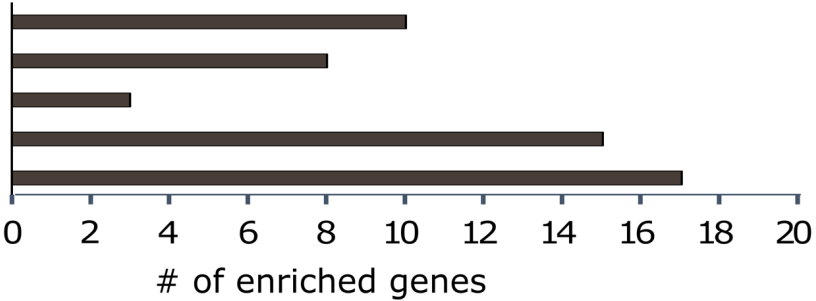

C

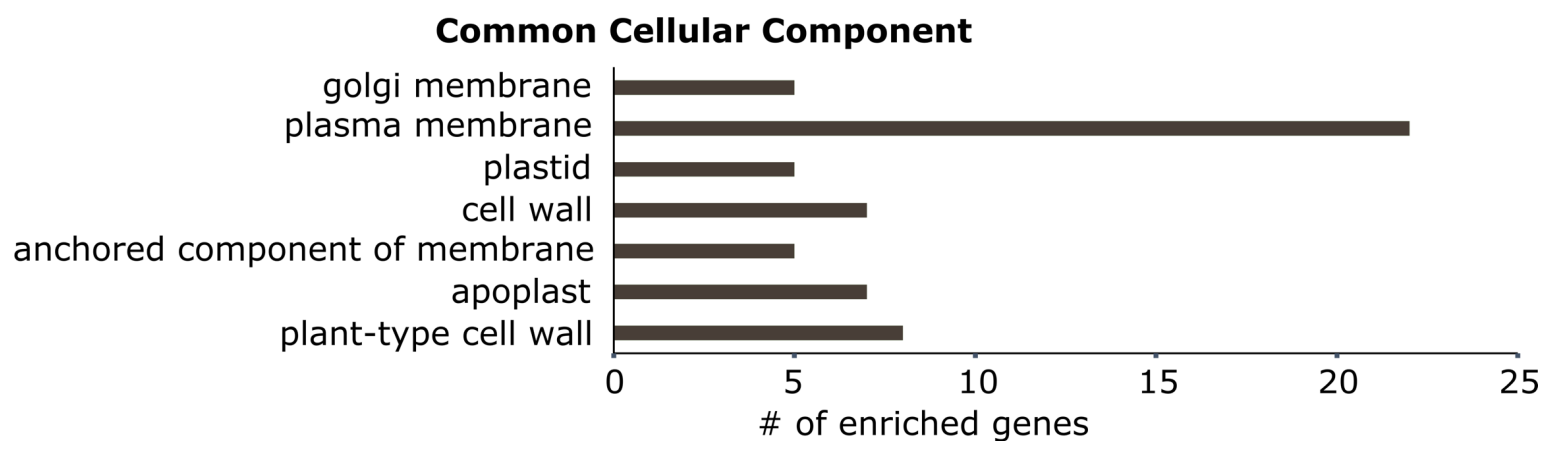

Figure 6 


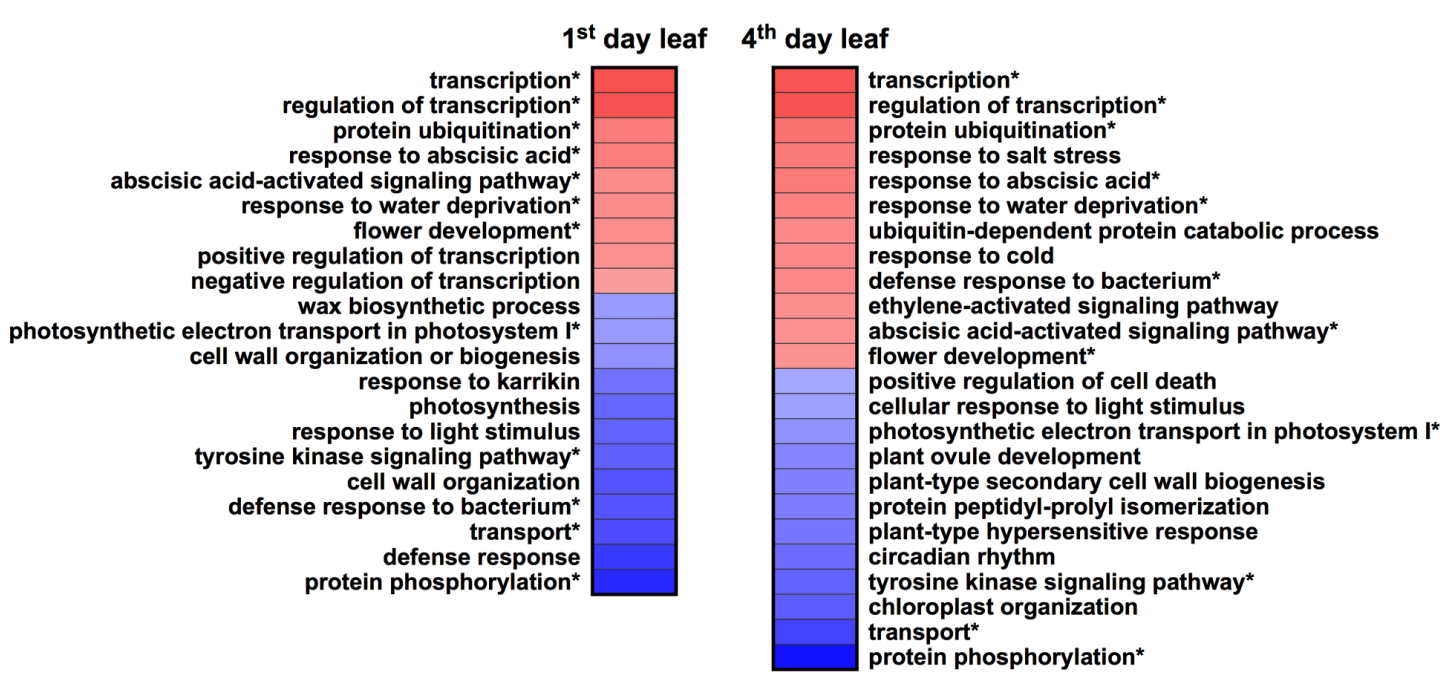

b

\section{$1^{\text {st }}$ day root $4^{\text {th }}$ day root}

transcription
regulation of transcription response to abscisic acid flower development response to salt stress ubiquitin-dependent protein catabolic process response to auxin response to water deprivation protein ubiquitination* response to heat response to cold protein transport negative regulation of transcription RNA splicing mRNA transport

covalent chromatin modification vegetative to reproductive phase transition of meristem eaf morphogenesis cellular response to DNA damage stimulus chaperone-media primary shoot apical meristem specification mRNA export from nucleus posttranscriptional tethering of RNA polymerase II nitrate transport vasculature development hydrogen peroxide catabolic process plant-type secondary cell wall biogenesis
response to chitin* response to chitin tyrosine kinase signaling pathway
cell wall organization cell wall organization

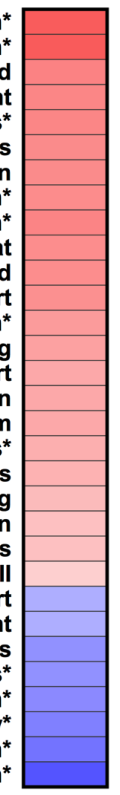

\# of enriched genes in each GO biological process

\begin{tabular}{|cc|ccc|}
\hline 64 & 8 & 0 & 8 & 64 \\
\hline & & \\
\hline & Down-regulated & Up-regulated
\end{tabular}

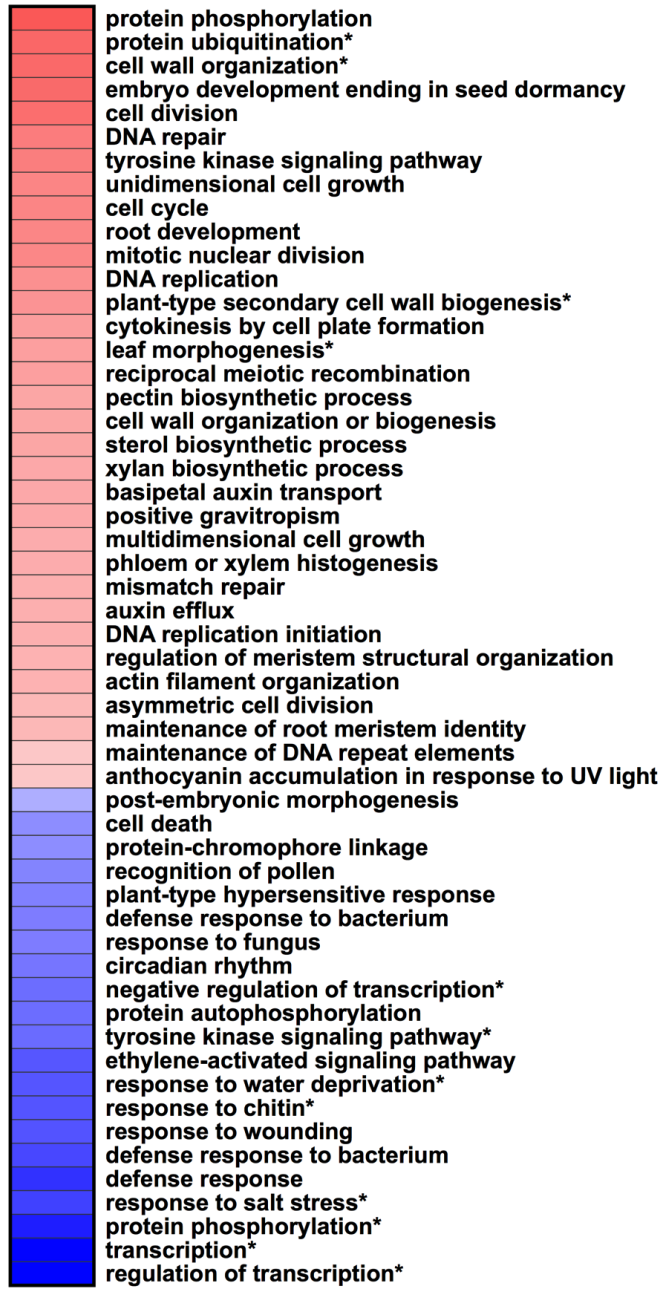

Figure 7 